\section{Update on microRNA as biomarkers of adrenocortical cancer: perspective on circulating microRNA}

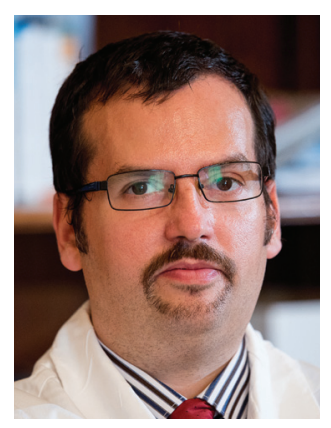

Peter lgaz

"MicroRNAs as biomarkers could be especially useful in tumors whose histological diagnosis is difficult, such as adrenocortical tumors.”

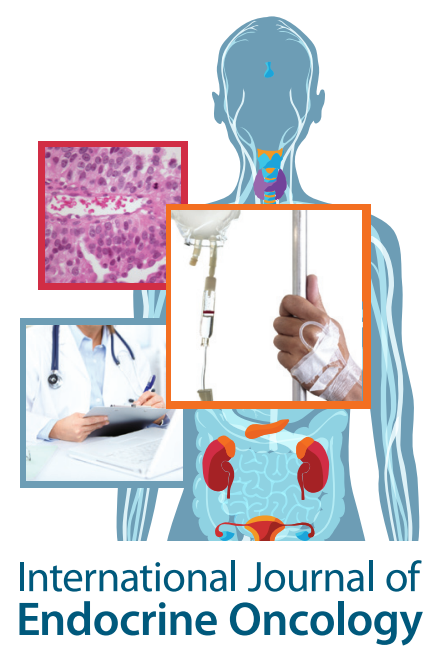

First draft submitted: 22 January 2017; Accepted for publication: 24 January 2017; Published online: 23 March 2017

Since my previous editorial on the potential of microRNAs as biomarkers of adrenocortical cancer (ACC) published in International Journal of Endocrine Oncology in 2015 [1], there have been some developments in the field. This short overview will focus on circulating microRNAs that may become applicable as minimally invasive biomarkers for diagnosis and prognosis, and even for therapy monitoring.

MicroRNAs are involved in the regulation of numerous physiological and pathophysiological processes including tumorigenesis, and numerous data underline their applicability as markers of malignancy and prognosis, and for tumor classification $[2,3]$. MicroRNAs as biomarkers could be especially useful in tumors whose histological diagnosis is difficult, such as adrenocortical tumors $[1,4]$. Although benign adrenocortical tumors are common, ACC is rare with an estimated incidence of $0.5-2$ cases/ million/year [5]. Unfortunately, there is no available preoperative blood-borne marker of malignancy for ACC, and biopsy is usually not performed due to the difficulties of histological diagnosis and fear for tumor spread [5]. At present, the suspicion for ACC is most often raised by imaging. Tissue microRNAs, for example, overexpressed $h s a-m i R-483-5 p$, hsa-miR-503 or underexpressed $h s a-m i R-195$ were shown to be promising markers of adrenocortical malignancy [4,6-7], but tissue microRNAs can be generally used in the postoperative setting.

Recent discoveries have shown that microRNAs are released from cells either via passive (e.g., necrosis, inflammation) or active secretion; active secretion of microRNAs occurs either in extracellular membrane vesicles (microvesicles, exosomes, apoptotic bodies) or in macromolecular complexes (e.g., complexed with Argonaute 2 protein or high density lipoprotein) [8]. Since microRNAs are involved in the regulation of gene expression forming part of the epigenetic machinery, extracellular microRNA profiles might convey valuable information on cellular functioning. The released microRNA profile is often different from

12nd Department of Medicine, Faculty of Medicine, Semmelweis University, H-1088 Budapest, Szentkirályi str. 46., Hungary; Tel./Fax: +36 1 2660816; igaz.peter@med.semmelweis-univ.hu 
"Given the major

difficulties in the diagnosis of ACC including the lack of an applicable preoperative blood marker of malignancy, and difficulties of imaging and histological analysis, circulating microRNAs might be envisaged as potential, novel minimally invasive markers of malignancy." the intracellular microRNA pool, however, the cellular mechanisms for active microRNA secretion are largely unknown [8]. Extracellular microRNAs can be found in body fluids including blood and they are remarkably stable [9].

Circulating microRNAs can thus be exploited as minimally invasive biomarkers, as a form of liquid biopsy. A huge body of research articles underlines the potential applicability of circulating microRNAs in the diagnosis of various diseases, for example, tumors, cardiovascular diseases, autoimmunity, etc $[10,11]$. The analysis of circulating microRNAs, however, is rather difficult that is in part related to their very low concentration (femtomolar range for individual microRNAs) [12], different analytic platforms used, lack of standards for normalization and reference genes [13].

Circulating microRNAs are also hypothesized to be involved in intercellular communication conveying epigenetic gene expression information [14]. By analyzing the profile of circulating microRNAs in healthy individuals, we have noted the relative overexpression of predominantly tumor suppressor microRNAs, and raised the hypothesis that circulating microRNAs might be involved in a tumor surveillance mechanism [15].

Given the major difficulties in the diagnosis of ACC including the lack of an applicable preoperative blood marker of malignancy, and difficulties of imaging and histological analysis [5], circulating microRNAs might be envisaged as potential, novel minimally invasive markers of malignancy.

Three studies have been published to date on the applicability of unfractionated human plasma or serum samples for ACC diagnosis $[13,16,17]$. The overexpression of circulating hsa-miR-483-5p has been described in ACC in all studies $[13,16,17]$ relative to benign adenomas, but Chabre et al. found its expression only in aggressive ACC and raised its potential applicability as a marker for shorter recurrence-free and overall survival along with underexpressed hsa-miR-195 [16]. In addition to hsa-miR-483-5p and $h$ sa-miR-195, some other circulating microRNAs have been described including underexpressed $h s a-m i R-335$ [16], and overexpressed hsa-miR-100, hsa-miR-181b, hsa-miR-184, hsa$m i R-210$ [13] and hsa-miR-34a [17]. These circulating microRNAs are thus promising markers of malignancy and hsa-miR-483-5p might even be exploited as a prognostic marker [16].
The sensitivity and specificity values of most of these microRNAs, however, do not seem to be high enough for clinical applicability at present. The AUC value for circulating hsa$m i R-483-5 p$ for differentiating adrenocortical adenomas and ACC has been 0.74 [17], however, much better sensitivity and specificity values have been described in the study of Chabre et al. for differentiating aggressive and nonaggressive ACC [16]. The best markers in our study $\left(\mathrm{dCT}_{\text {hsa-miR-210 }}-\mathrm{dCT}_{\text {hsa-miR-181b }}\right.$ and $\mathrm{dCT}_{\text {hsa-miR-100 }} /$ $\mathrm{dCT}_{\text {hsa-miR-181b }}$ ) turned out to have AUC values of 0.85 and 0.87 , respectively [13]. Studies on larger cohorts of patients are thus needed to establish the applicability of circulating microRNAs for clinical diagnosis.

In a most novel study, we have investigated extracellular vesicle-associated microRNAs hypothesizing that actively secreted microRNAs might be more specific for diagnosis than microRNAs from unfractionated plasma. Unfractionated plasma contains cellular debris, whereas the content of extracellular vesicles results from active secretion and thus might be directly linked to cellular activity. In fact, we have found much fewer significantly differentially expressed microRNAs in extracellular vesicle preparations than in unfractionated plasma that might also confirm our hypothesis that extracellular vesicles could be more specific as a source of microRNAs [Perge et al. Evaluation AND DIAGNOSTIC POTENTIAL OF CIRCULATING EXTRACELLULAR VESICLE-ASSOCIATED MICRORNAS IN ADRENOCORTICAL TUMORS, SUBMITTED].

A further potential clinical application of circulating microRNAs could be disease-progression monitoring. We have studied circulating microRNA markers in two ACC xenograft models: in an NCI-H295R xenograft model, we have noted that treatment with 9-cis retinoic acid significantly decreased circulating hsa-miR-483-5p expression [18]; in contrast, circulating hsa-miR483-5p levels have not been affected by shortterm cytotoxic liposomal-EDP-M (etoposidedoxorubicin-cisplatin-mitotane) chemotherapy in either SW-13 or SJ-ACC3 (a pediatric ACC model) xenografts [19]. Another microRNA, hsa$m i R-210$ that is a major hypoxamiR [20] and was found to be overexpressed as tissue microRNA in ACC, as well [7], turned out to display elevated plasma levels after liposomal EDP-M chemotherapy in the SW-13 model [19]. Considering the lack of suitable blood-borne markers of ACC progression at present, if validated in the human 
setting, these circulating microRNAs might be of considerable clinical relevance.

In conclusion, circulating microRNAs might be promising minimally invasive biomarkers of ACC and as markers of malignancy, prognosis and even applicable for monitoring the efficacy of anticancer treatment. More studies are needed, however, on larger scale cohorts with uniform methodology to be able to reliably assess the clinical utility of circulating microRNAs in ACC diagnosis.

\section{Future perspective}

Circulating microRNAs could be exploited as minimally invasive markers of adrenocortical malignancy. MicroRNAs in extracellular vesicles might turn out to be more specific than unfractionated plasma samples. In addition to being used as markers of malignancy, circulating microRNAs might be envisaged as markers of prognosis or for monitoring disease progression, however, further large-scale studies are needed to establish their utility in clinical diagnosis.

\section{Financial \& competing interests disclosure}

The study has been supported by a grant from the Hungarian National Research, Development and Innovation Office (NKFIH K115398) to P Igaz. The author has no other relevant affiliations or financial involvement with any organization or entity with a financial interest in or financial conflict with the subject matter or materials discussed in the manuscript apart from those disclosed.

No writing assistance was utilized in the production of this manuscript.

\author{
“...circulating microRNAs \\ might be promising \\ minimally invasive \\ biomarkers of \\ adrenocortical cancer and \\ as markers of malignancy, \\ prognosis and even \\ applicable for monitoring \\ the efficacy of anticancer \\ treatment."
}

\section{References}

1 Igaz P. Can microRNA be used as a biomarker in adrenocortical cancer? Int. J. Endocr. Oncol. 2(2), 101-103 (2015).

2 Lu J, Getz G, Miska EA et al. MicroRNA expression profiles classify human cancers. Nature 435(7043), 834-838 (2005).

3 Iorio MV, Croce CM. microRNA involvement in human cancer. Carcinogenesis 33(6), 1126-1133 (2012).

4 Igaz P, Igaz I, Nagy Z et al. MicroRNAs in adrenal tumors: relevance for pathogenesis, diagnosis, and therapy. Cell. Mol. Life Sci. 72(3), 417-428 (2015).

5 Fassnacht M, Kroiss M, Allolio B. Update in adrenocortical carcinoma. J. Clin. Endocrinol. Metab. 98(12), 4551-4564 (2013).

6 Soon PS, Tacon LJ, Gill AJ et al. miR-195 and miR-483-5p Identified as predictors of poor prognosis in adrenocortical cancer. Clin. Cancer Res. 15(24), 7684-7692 (2009).

7 Tombol Z, Szabo PM, Molnar V et al. Integrative molecular bioinformatics study of human adrenocortical tumors: microRNA, tissue-specific target prediction, and pathway analysis. Endocr. Relat. Cancer 16(3), 895-906 (2009).
8 Turchinovich A, Tonevitsky AG, Burwinkel B. Extracellular miRNA: a collision of two paradigms. Trends Biochem. Sci. 41(10), 883-892 (2016).

9 Weber JA, Baxter DH, Zhang $S$ et al. The microRNA spectrum in 12 body fluids. Clin. Chem. 56(11), 1733-1741 (2010).

10 Schwarzenbach H, Nishida N, Calin GA, Pantel K. Clinical relevance of circulating cell-free microRNAs in cancer. Nat. Rev. Clin. Oncol. 11(3), 145-156 (2014).

11 Reid G, Kirschner MB, van Zandwijk N. Circulating microRNAs: association with disease and potential use as biomarkers. Crit. Rev. Oncol. Hematol. 80(2), 193-208 (2011).

12 Williams Z, Ben-Dov IZ, Elias R et al. Comprehensive profiling of circulating microRNA via small RNA sequencing of cDNA libraries reveals biomarker potential and limitations. Proc. Natl Acad. Sci. USA. 110(11), 4255-4260 (2013).

13 Szabo DR, Luconi M, Szabo PM et al. Analysis of circulating microRNAs in adrenocortical tumors. Lab. Invest. 94(3), 331-339 (2014).

14 Perge P, Nagy Z, Decmann A, Igaz I, Igaz P. Potential relevance of microRNAs in inter-species epigenetic communication, and implications for disease pathogenesis. $R N A$ Biol. doi:10.1080/15476286.2016.1251001 (2016) (Epub ahead of print).

15 Igaz I, Igaz P. Tumor surveillance by circulating microRNAs: a hypothesis. Cell. Mol. Life Sci. 71(21), 4081-4087 (2014).

16 Chabre O, Libe R, Assie G et al. Serum miR-483-5p and miR-195 are predictive of recurrence risk in adrenocortical cancer patients. Endocr. Relat. Cancer 20(4), 579-594 (2013).

17 Patel D, Boufraqech M, Jain M et al. MiR-34a and miR-483-5p are candidate serum biomarkers for adrenocortical tumors. Surgery 154(6), 1224-1228 (2013).

18 Nagy Z, Baghy K, Hunyadi-Gulyas E et al. Evaluation of 9-cis retinoic acid and mitotane as antitumoral agents in an adrenocortical xenograft model. Am. J. Cancer Res. 5(12), 3645-3658 (2015).

19 Jung S, Nagy Z, Fassnacht M et al. Preclinical progress and first translational steps for a liposomal chemotherapy protocol against adrenocortical carcinoma. Endocr. Relat. Cancer 23(10), 825-837 (2016).

20 Devlin C, Greco S, Martelli F, Ivan M. miR-210: more than a silent player in hypoxia. IUBMB Life 63(2), 94-100 (2011). 